\title{
Neubestimmung des Verhältnisses der Molekulargewichte von Kaliumchlorat und Kaliumchlorid.
}

\author{
Von \\ Arthur Stähler und Friedrich Meyer.
}

Mit 4 Figuren im Text.

\section{Geschichtliches.}

Die Atomgewichte der meisten Grundstoffe gründen sich in experimenteller Hinsicht auf die Atomgewichte des Chlors und Silbers. Der Grund hierfür liegt darin, dals die Chloride der Elemente meist leicht zugänglich sind und sich glatt mit Silbernitrat in Silberchlorid überführen lassen, das ebenfalls sehr rein erhalten werden kann. Aus dieser Tatsache ergibt sich die wichtige Forderung, das Verhältnis der Atomgewichte von Chlor und Silber sowohl zueinander als zu dem des Sauerstoffs, der Grundeinheit, mit möglichst grofser Genauigkeit festzustellen.

Der erste Teil dieser Aufgabe, die Bestimmung des Verhältnisses der Atomgewichte von Chlor und Silber zueinander, kann durch die ausgezeichneten Arbeiten von T. W. RICEARDS and seinen Mitarbeitern nach dem Stande unserer heutigen Experimentierkunst als gelöst betrachtet werden. Dagegen lagen für das Verhältnis des A tomgewichtes des Sauerstoffs zu dem des Chlors, und damit indirekt auch zu dem des Silbers bis vor kurzem nur ältere Arbeiten über die quantitative Zersetzung der Chlorate vor, unter denen die von STAS ausgeführten als die besten galten.

Als Ausgangsmaterial wurde bei diesen Untersuchungen meist das leicht umzukristallisierende Kaliumchlorat benutzt. Die ersten guten Analysen dieser Verbindung veröffentlichte Berzelius. ${ }^{1}$ Er zersetzte trockenes Kaliumchlorat durch Erhitzen und suchte den hauptsächlichsten Fehler seiner Vorgänger, die auf die etwaige Verflüchtigung des während der Operation entstehenden Kaliumchlorids

1 Pogg. Ann. 8 (1826), 17. Die früheren Arbeiten haben nur geschichtliches Interesse. 
keine Rücksicht genommen hatten, durch verschiedene, hier nicht näher wiederzugebende Kunstgriffe zu vermeiden.

Die nächste Analysenreihe führt PENNY ${ }^{1}$ aus, der das Salz mit starker Salzsäure eindampfte und das zurückbleibende Kaliumchlorid wog.

Pelodze, ${ }^{2}$ Marignac, ${ }^{3}$ Gerhardt ${ }^{4}$ und Madmene ${ }^{5}$ zerlegten das Chlorat nach dem Vorgang von Benzeurus durch Erhitzen und wandten ebenfalls verschiedene Mittel zur Ermittelung der dabei verflüchtigten und vom Gasstrome mitgerissenen Substanzmenge an.

Als letzter folgte STAs ${ }^{6}$ mit zwei verschiedenen Versuchsreihen. Er verwandelte das Kaliumchlorat sowohl durch blofses Erhitzen, als auch nach der Methode von PENwy durch Behandeln mit starker Salzsäure in das Chlorid. STas traf bei seiner Untersuchung alle erdenklichen Vorsichtsmafsregeln, so dafs ein Irrtum kaum möglich erschien.

Die in neuester Zeit insbesondere von RICHARDs und seinen Schülern über Atomgewichte ausgeführten Arbeiten haben nun trotz aller Bewunderung, die man den Methoden von STAS zollen mufs,

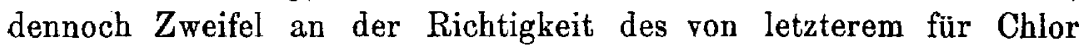
gefundenen Wertes entstehen lassen. Diesem Umstande sind auch die Versuche zuzuschreiben, die man neuerdings angestellt hat, das Atomgewicht des Chlors auf anderem Wege zu finden.

Hier ist zunächst zu erwähnen, dals man, namentlich auf Anregung von D. BERTHELot ${ }^{7}$ versucht hat, das Avogadro sche Prinzip für die Atomgewichtsbestimmung in der Weise nutzbar zu machen, dafs man unter Berücksichtigung der VAN DER WAALS schen Gleichung exakte Gasdichtebestimmungen ausführte. Gerade für Chlor gibt jedoch, wegen dessen leichter Kondensierbarkeit, diese sonst recht brauchbare Methode etwas unsichere Werte.

Ferner haben, um das Problem zu lösen, Gure und Fudss ${ }^{8}$ die vollständige Analyse von Nitrosylchlorid ausgeführt, sowie T. W. RICHaRdos und H. H. WILLARD ${ }^{y}$ das Verbältnis von Lithiumchlorid

1 Phil. Trans. 1839, 20.

2 Compt. rend. 15 (1842), 959.

3 Ann. Chim. Pharm. 44 (1842), 18.

4 Compt. rend. 21 (1845), 1280.

5 Ann. Chim. Phys. [3] 18 (1846), 80.

6 Oeuvres Compl. 1, 395-405.

7 Journ. Phys. [3] 8 (1899), 263.

${ }^{8}$ Journ. Chim. Phys. (6 (1908), 732.

9 Z. anorg. Chem. 66 (1910), 229. 
zu Lithiumperchlorat sowie von Lithiumchlorid zu Silber und silberchlorid bestimmt.

Trotz der grofsen Exaktheit, mit der die Messungen durchgeführt wurden, weichen die Resultate dieser neueren Arbeiten zwar nicht viel, aber doch soweit voneinander ab, dals das Atomgewicht des Chlors noch nicht als genügend festgelegt gelten kann.

Die im folgenden mitgeteilte Arbeit ist im Sommer 1907 auf Veranlassung des damaligen Austauschprofessors 'T. W. Richands von StäHLER begonnen und nach einigen Unterbrechungen von Stähler und Meyer zu Ende geführt worden. Der Zweck der Untersuchung war, durch eine neue exakte Bestimmung des Verhältnisses $\mathrm{KClO}_{3}: \mathrm{KCl}$ eine Entscheidung darüber zu bringen, welcher unter den verschiedenen Werten dem Chlor zukommt, und zugleich eine kritische Revision der oben erwähnten Arbeit von STas zu liefern.

\section{Allgemeiner Arbeitsweg.}

Die Fortschritte der modernen Atomgewichtsforscher gegenüber den Arbeiten von STAS und seinen Zeitgenossen sind zum Teil der vorsichtigen Auswahl des Gefäls- und Anaiysenmateriales, zum Teil auch der Beachtung einer gröfseren Zahl, den älteren Forschern unbekannter physikalisch-chemischer Vorgänge zuzuschreiben.

Dies ist ausführlich in einem Vortrag „Neuere Untersuchungen über Atomgewichte" von 'T. W. Richards im Jahre 1907 in der Deutschen Chemischen Gesellschaft behandelt worden, ${ }^{1}$ so dals hier nicht mehr darauf eingegangen werden soll.

In vorliegender Untersuchung wurde Kaliumchlorat unter Verwendung der Zentrifuge mebrfach umkristallisiert und durch Salzsäure in das Chlorid rerwandelt.

Diese Methode hat vor der Zerlegung des Chlorats durch Erhitzen des trockenen Salzes einen grofsen Vorzug. Hierbei sind nämlich Verluste an Material fast unvermeidlich, da das entstehende Kaliumchlorid schon unterhalb Rotglut etwas flüchtig ist und auch das geschmolzene Chlorat-Chloridgemenge durch den entweichenden Sauerstoff zerstäubt und spurenweise in Form eines äufserst feinen, kaum kondensierbaren Nebels mit fortgeführt wird.

Stas hatte die Zersetzung des Chlorats derart vorgenommen, dafs er das Salz in einem Glaskolben schmolz, wog, mit reinem

\footnotetext{
1 Ber. 1907, 2767.
} 
Wasser überschichtete und dann unter Kühlung mit Eis im Salzsäurestrom zersetzte. Die gasförmigen Reaktionsprodukte leitete er durch gläserne, mit Wasser beschickte Flaschen, um etwa vom Gasstrom mitgerissene Substanz zurückzuhalten. War die Zersetzung, die sich so vollzog, dafs zum Schlufs etwa die Hälfte des entstandenen Kaliumchlorids in ungelöster Form vorlag, beendet, so verdampfte er den Inhalt des Kolbens im Luftstrome zur Trockne. STas konnte, da er mit Glasgefälsen arbeitete, das zurückgebliebene Kaliumchlorid nicht schmelzen. Er setzte daher zur Entfernung der noch stets darin vorhandenen Salzsäure eine vorher genau gewogene Menge Chlorat hinzu und erhitzte das Gemisch. Dabei wurde das neu hinzugefügte Chlorat zum Teil durch die noch vorhandene Salzsäure, zum Teil durch dio Wirkung der Hitze in Chlorid übergeführt.

Bei kritischer Betrachtung dieser Arbeitsmethode vom Standpunkte der modernen Atomgewichtsforschung erkennt man sofort einige mögliche Fehlerquellen. Stas hat zwar das Chlorat vor der Wägung und vor der Zersetzung durch Salzsäure geschmolzen, wie das neuerdings bei der exakten Bestimmung des Gewichtes von Silzen mit Recht gefordert wird, jedoch weniger in der Absicht, das inkludierte Wasser zu entfernen, als vielmehr, um der beim Zersetzen des kristallisierten Salzes mit Chlorwasserstoff bestehenden Gefahr einer Explosion vorzubeugen. Während der Versuche zur Zersetzung des trockenen Chlorats durch blofses Erhitzen hat er sich damit begnügt, das Salz bei etwa $350^{\circ}$ im Luftstrome vor der Wägung zu trocknen.

Bei einer Nachprüfung fanden wir, dafs sowohl das Trocknen bei $350^{\circ}$ als auch das Schmelzen des Kaliumchlorats mit darauffolgender Wägung fehlerhaft war. Dieser Irrtum ist vielleicht der schwerste, den STAS bei seiner Arbeit begangen hat. STAS gibt an, dals man reines Kaliumchlorat unzersetzt schmelzen könnte, und stützt seine Angabe darauf, dals das bei $350^{\circ}$ getrocknete Salz beim Schmelzen gewichtskonstant bleibt. Tatsächlich zeigt nach unseren Beobachtungen ein bei dieser Temperatur getrocknetes Salz nach dem Schmelzen nur eine äufserst geringe Gewichtsabnabme, das Chlorat ist aber trotzdem schon durch das Trocknen unter diesen Umständen weitgehend zersetzt worden, da es eine beträchtliche Menge Cl-Ion enthält (s. u.).

Der zweite Fehler von Sras war der, dafs er nach beendeter Zersetzung des Chlorats durch Salzsäure einen Teil des Kalium. chlorids ungelöst zurückbehielt; denn dadurch entstand die Gefabr, 
dafs von den Kristallen eingeschlossenes Chlorat der Zersetzung entgehen könnte.

STaS vermochte wegen des ihm zur Verfügung stehenden Gefärsmateriales das Kaliumchlorid nicht zu schmelzen und war daher gezwungen, jenen Kunstgriff anzuwenden, durch Zugabe von Kaliumchlorat (etwa 2-5\% des Chlorids) die Salzsäure, die er durch Erhitzen nicht vertreiben konnte, zu entfernen. Bei der geringen Menge Chlorat und der relativ niedrigen Temperatur, die er anwenden konnte, lief er Gefahr, dals sein Kaliumchlorid zum Schlufs neben Spuren Wasser immer noch Salzsäure und walrscheinlich auch Chlorat enthielt. Wir konnten durch Verwendung von Quarzglas, das sich widerstandsfähig gegen geschmolzenes Kaliumchlorid erwies, diese Fehlerquelle vermeiden.

In aller Kürze ist demnach unsere von der STAsschen abweichende Arbeitsweise die folgende:

Reinstes, kristallisiertes Kaliumchlorat wurde bei gewöhnlicher Temperatur in einem geeigneten aus Quarzglas hergestellten Zersetzungsgefäls getrocknet und gewogen, darauf durch Schmelzen des Chlorats im trocknen Luftstrome das inkludierte Wasser ausgetrieben und letzteres in einem Phosphorpentoxydrohr aufgefangen. Das Gewicht des kristallisierten Kaliumchlorats, vermindert um die Gewichtszunahme des Phosphorpentoxydrobres, ergab das Gewicht des wasserfreien Salzes in Luft. Das Zersetzungskölbchen wurde nun mit der nötigen Menge Wasser beschickt und die Reduktion des Chlorats durch Einleiten von reinem Salzsäuregas vorgenommen. Die gasförmigen Zersetzungsprodukte wurden nicht wie bei STAS durch Waschen mit Wasser von der mitgerissenen Substanz befreit, sondern wir kondensierten mit Hilfe von flüssiger Luft sämtliche aus dem Gefäls entweichenden Gase in zwei Vorlagen aus Quarzglas, so dafs während des ganzen Prozesses keine merklichen Mengen Gas aus dem Apparat entweichen konnten, und daher alle mitgerissene Substanz sich in den Vorlagen finden mulste. Die salzsäurehaltige Chloridlösung wurde wie bei STAS im Luftstrom unterhalb der Siedetemperatur eingedampft. Das zurückbleibende Kaliumchlorid wurde von uns jedoch in einer Stickstoffatmosphäre zur Entfernung von Wasser und Salzsäure geschmolzen. Die sehr geringe, in den Vorlagen enthaltene Substanzmasse wurde, aus Gründen, die weiter unten näher angegeben werden, nach dem Verdampfen der Salzsäure durch Behandlung mit Überchlorsäure in 
Kaliumperchlorat übergeführt und die daraus bestimmte Menge Chlorid zu der Hauptmenge gerechnet.

Die folgende Tabelle gibt alle Daten einer wirklich ausgeführten Bestimmung an:

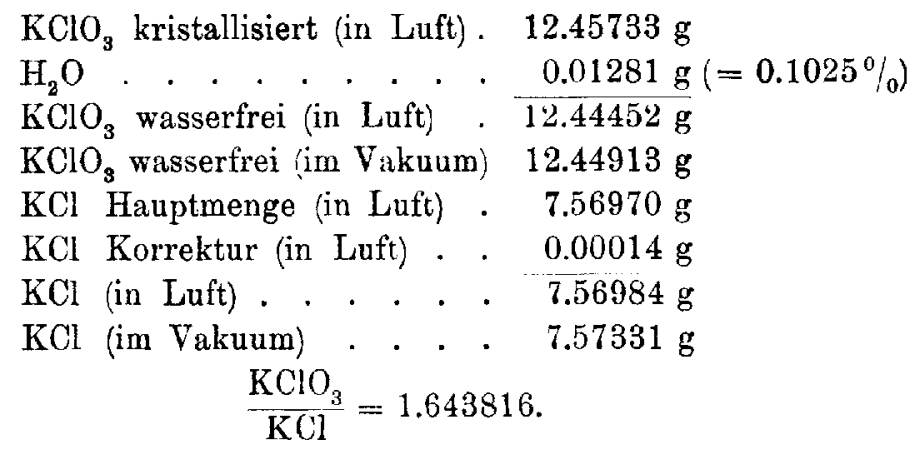

Experimentelles.

\section{Ausgangsmaterial.}

\section{Herstellung reinsten Wassers.}

Destilliertes Wasser (aus der Destillationsanlage des Instituts) wurde mit alkalischer Permanganatlösung, nach einem von Professor Brauner.Prag privatim gemachten Vorschlage, mehrere Tage im Sonnenlicht stehen gelassen und daun aus einem Richardskolben unter Verwendung eines Glaskühlers abdestilliert. Dieses Wasser war chlorfrei und konnte zu nephelometrischen Beobachtungen Verwendung finden. Da jedoch etwa vorhandenes Ammoniak das Wasser zur Kristallisation von Kaliumchlorat noch nicht geeignet erscheinen liefs, wurde es einer weiteren Destillation unter Zugabe einer kleinen Menge Kaliumbisulfat unterworfen, wobei, um das Wasser möglichst kieselsäurefrei zu halten, ein Kühler aus Zinn zur Verwendung gelangte. Damit nun auch die letzten mit dem Dampfstrome etwa herübergerissenen Teilchen von Kaliumbisulfat und Kieselsäure entfernt würden, wurde dieses Wasser noch einmal für sich durch einen Platinkübler destilliert. Nachdem der Destillationsapparat mehrere Stunden in Betrieb war, hinterliefsen $200 \mathrm{ccm}$ des so gewonnenen Wassers nach dem Verdampfen in einer blanken Platinschale weder einen sichtbaren noch wägbaren Rückstand.

\section{Kaliumehlorat.}

Wir prüften zunächst die Frage, ob Kaliumchlorat frei von Kaliumchlorid bzw. $\mathrm{Cl}^{\prime}$ hergestellt werden kann. Dies war neuer- 
dings von GLYE $^{1}$ bestritten worden. Nach letzterem soll in $1 \mathrm{~g}$ Kaliumchlorat stets $0.27 \mathrm{mg}$ Kaliumchlorid enthalten sein.

$\mathrm{Zu}$ diesem $\mathrm{Zweck}$ haben wir mehrfach umkristallisiertes Kaliumchlorat einigen weiteren Kristallisationen ${ }^{2}$ unterworfen und hierbei die grölste Vorsicht angewandt, um jede Zersetzung oder Verunreinigung des Chlorats zu vermeiden.

So wurde der Einschlufs von Kieselsäure durch ausschlielsliche Verwendung von Platingefälsen und von reinstem Wasser verhindert. Als Kristallisationsgefäls diente eine grofse Platinschale von ca. 2 I Inhalt, als Rührer ein Spatel aus demselben Metall. Ein elektrisch geheiztes Bad (vgl. S. 387) lieferte die zum Lösen nötige Wärme, wodurch einer Verunreinigung des Chlorats durch die Verbrennungsprodukte (z. B. schweflige Säure) eines Heizmaterials vorgebeugt war. Zum Schutze gegen Staub wurde ein Schutztrichter nach V. Merer über die Schale gehängt. Der Raum selbst wurde von allen schädlichen Gasen, vor allem von Salzsäuredämpfen, freigehalten.

Bei diesen ersten Kristallisationen wurde etwas mehr Wasser, als zum Lösen des Salzes bei $100^{\circ}$ erforderlich war, verwendet, und die Flüssigkeit so lange abgedampft bis sich eben eine Salzhaut bildete. Dabei stieg die Temperatur der Lösung in der Regel auf $102-103^{\circ} \mathrm{C}$. War dieser Moment eingetreten, so liefs man die Lösung unter einer Glocke sich abkühlen. Nach beendeter Kristallisation wurde die Mutterlauge durch zwei mit Platinkonen versehene Platintrichter abgegossen und ein Teil daron im Nephelometer auf Chlorion untersucht, die Kristallmasse auf dem Platintrichter gesammelt und auf der Zentrifuge soweit als möglich von der Mutterlauge befreit. Ausgewaschen wurde bei diesen Versuchen nicht.

Im folgenden einige Zahlen über den Gehalt an Chlorion der Mutterlaugen:

Die angewandte Substanz enthielt . . pro $1 \mathrm{~g} \mathrm{ca} 0.015 \mathrm{mg} \mathrm{Cl}$

Die Mutterlauge der 1. Fraktion. . . " $1 \mathrm{~g}, 0.0050, \mathrm{Cl}^{\prime}$

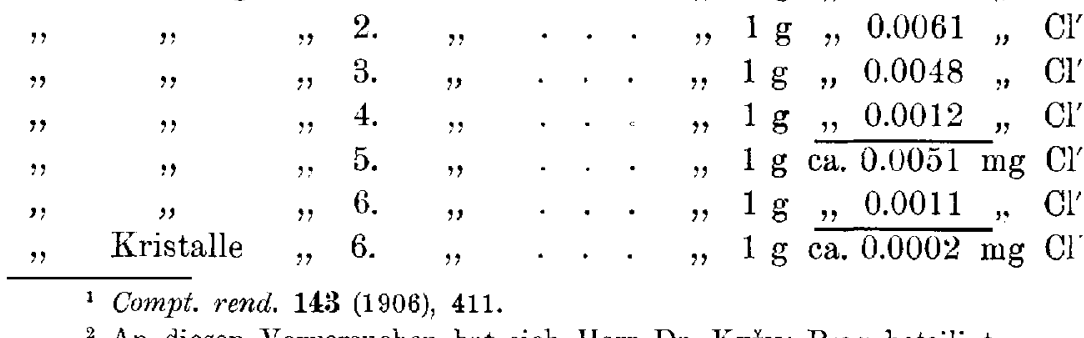

${ }^{2}$ An diesen Vorversuchen hat sich Herr Dr. Kužra-Prag beteiligt. 
Bei der 4. Fraktion, die auffallend wenig $\mathrm{Cl}^{\prime}$ enthielt, war die Lösung nur auf ca. $80^{\circ}$ statt auf $100^{\circ}$ erhitzt worden, da versehentlich zu viel Wasser zu dem Salz gegeben war und die Zeit zum Eindampfen fehlte.

Bei der Fraktion 6 war deshalb wissentlich soviel Wasser verwendet worden, dafs eine gesättigte Lösung bei $80^{\circ}$ entstand, um die Vermutung, ob der Gehalt an Kaliumchlorid von der Lösungstemperatur abhänge, zu prüfen. Der Versuch hat diese Vermutung bestätigt und gleichzeitig den Weg gezeigt, auf dem ein Kalinmchlorat höchster Reinheit zu erlangen ist. Aus obigem Befunde geht im übrigen hervor, dafs Kaliumchlorat mit einer sehr kleinen konstanten Menge Kaliumchlorid aus der Lösung auskristallisiert, wenn man bei konstanter Temperatur arbeitet. Diese Verunreinigung ist aber so verschwindend klein, dafs den Angaben Gryes ein Versehen zugrunde liegen mufs. Vielleicht ist letzterer durch irgendwelche Nebenumstände irregeleitet worden; so bewirkt z. B. die Verwendung einer nur geringe Mengen nitroser Produlte enthaltenden Salpetersäure beim Nachweis des $\mathrm{Cl}^{\prime}$ einen erheblichen Fehler, da durch niedere Stickoxyde das Chlorat reduziert wird.

Das von STAS hergestellte Kaliumchlorat war zwar nicht ron absoluter, so doch von einer Reinheit, die selbst für derartig präzise Bestimmungen als ausreichend zu bezeichnen ist; denn das nach seinen Angaben bei $100^{\circ}$ hergestellte Salz enthält auf $100 \mathrm{~g}$ Kaliumchlorat erst ca. $0.3-0.4 \mathrm{mg}$ Kaliumchlorid. Dieser Cl'Gehalt ist so gering, dals er nur noch mit Hilfe des Nephelometers feststellbar ist.

Die nächsten Versuche galten der Prüfung der Angabe von Stas über die Beständigkeit des Kaliumchlorats beim Schmelzen. Die Substanz wurde auf die verschiedenste Weise getrocknet, im Vakuum ïber Phosphorpentoxyd (was iubrigens wegen der möglichen Entwickelung von Phosphorwasserstoffen, die, wenn auch eine schwache, so doch merkliche Verumreinigung des Kaliumchlorats verursachen, nicht zu empfehlen ist), bei $100^{\circ}$ und schlielslich durch längeres Aufbewahren im Exsikkator über festem Kaliumbydrat.

STaS hat nämlich das Salz gewogen, geschmolzen und nach dem Wiederwägen keine Zersetzung feststellen können. Es war zwar von vornherein sehr unwahrscheinlich, dals sich das Salz, das wir wie STAS bei $100^{\circ}$ getrocknet hatten, obne Gewichtsverlust würde schmelzen lassen; trotzdem wurden dahingeliende Versuche gemacht unter der Annahme, dals Kaliumchlorat das inkludierte Wasser. 
vielleicht besonders leicht abgebe und bei intensivem Trocknen die Substanz tatsächlich mit keinem oder nur sehr geringem Gewichtsverlust schmelzbar sei. Diese Annahme bestätigte sich nicht; denn das geschmolzene Salz zeigte einen Gewichtsverlust von erheblich über $0.1 \%$ und war zudem stark zersetzt. Dabei stellte sich noch die Eigentümlichkeit heraus, dafs die Zersetzung um so geringer war, je schneller das Schmelzen erfolgte. So ergab ein Versuch im Quarzreagenzglas über freier Flamme den günstigsten Wert, etwa $0.04 \mathrm{mg} \mathrm{KCl}$ pro $1 \mathrm{~g}$ Substanz, entsprechend einem Sauerstoffverlust von ca. $0.02 \mathrm{mg}$, einem Wert, der bei langsamerem Erhitzen oder auch bei Anwendung gröfserer Mengen nicht annähernd erreicht wurde. Bei schnellem Schmelzen von etwa $10-12 \mathrm{~g}$ Kaliumchlorat mit dem elektrisch geheizten Luftbad in dem unten zu beschreibenden Zersetzungsapparat bilden sich für jedes Gramm Chlorat ca. $0.4 \mathrm{mg}$ Chlorid, also etwa das Zehnfache des vorhin erwähnten günstigsten Wertes.

Bei einer nochmaligen Durchsicht der Stas schen Arbeit fanden wir dann bei der Erklärung der Ausführung der Zersetzung des Chlorats durch Erhitzen die Angabe: „Das Salz wurde, bevor man es in den Apparat füllte, mehrere Stunden im trockenen Luftstrome, bis nahe an seinen Schmelzpunkt erhitzt, getrocknet." Diese Bemerkung von Sras, die bei seiner Vorschrift für die Herstellung von reinem Kaliumchlorat (danach sollte es bei $100^{\circ}$ in Luftstrome ge. trocknet werden) fehlt, macht seinen Befund über die Zersetzung des Kaliumachlorats beim Schmelzen erklärlich, offenbart aber auch zugleich den schwersten Fehler der Stasschen Arbeit. Ein bei derartig hoher Temperatur (etwa $350^{\circ}$ ) getrocknetes Salz (die Hauptmenge des Wassers entweicht aus dem Kaliumchlorat bei einer Badtemperatur von etwa $200-250^{\circ}$ ) zeigt nämlich tatsächlich beim Schmelzen nur einen sehr geringen Gewichtsverlust. Dafs in dem festen Chlorat schon Zersetzung hätte eingetreten sein können, scheint STAS übersehen zu haben; denn er gibt nirgends an, dals or die Substanz daraufhin untersucht habe.

Da aus unseren Schmelzversuchen hervorging, dafs das eingeschlossene Wasser entsprechend seiner Menge und der Zeitdauer seiner Einwirkung, in hervorragender Weise an der Zersetzung des Chlorats beim Schmelzen beteiligt ist, so war zu erwarten, dals das bei $350^{\circ}$ getrocknete Salz weit mehr Kaliumchlorid enthalten würde als das schnell geschmolzene. Tatsächlich ergab der Versuch eine so weitgehende Zersetzung, dafs der Gehalt an Chlorion einer bei 
Zimmertemperatur gesättigten Lösung im Nephelometer wegen der Dichte des Niederschlages nicht mehr bestimmt werden konnte.

Es stellte sich hier der Neubestimmung des Verhältnisses $\underset{\overline{\mathrm{KCl}}}{\mathrm{KClO}_{3}}$ eine erhebliche Schwierigkeit entgegen, da das genaue Gewicht des Kaliumchlorats nicht wie bei anderen Salzen durch eine nach dem Schmelzen erfolgende Wägung festgestellt werden konnte. Eine Bestimmung des Gehaltes an Chlorion des geschmolzenen Salzes hätte nicht viel geholfen, da wahrscheinlich eine unbekannte Menge Kaliumperchlorat, deren Bestimmung äufserst schwierig gewesen wäre und zudem durch die Zahl der notwendigen Korrekturen den Wert der Bestimmung ganz erheblich herabgedrückt hätte, beim Scbmelzen entstand. Der einzig mögliche Ausweg war, dafs das bei Zimmertemperatur getrocknete $\mathrm{Salz}$ gewogen und das inkludierte Wasser durch Austreiben und gesondertes Auffangen bestimmt wurde. Die Differenz der Werte $\mathrm{KClO}_{3}$ krist. $\mathrm{H}_{2} \mathrm{O}$ gab dann mit sehr kleinem Fehler das Gewicht des wasserfreien Kaliumchlorats in Luft.

Nachdem die Apparatur, mit der die Zersetzung ausgeführt werden sollte, eine dem neuen $Z$ wecke ent-

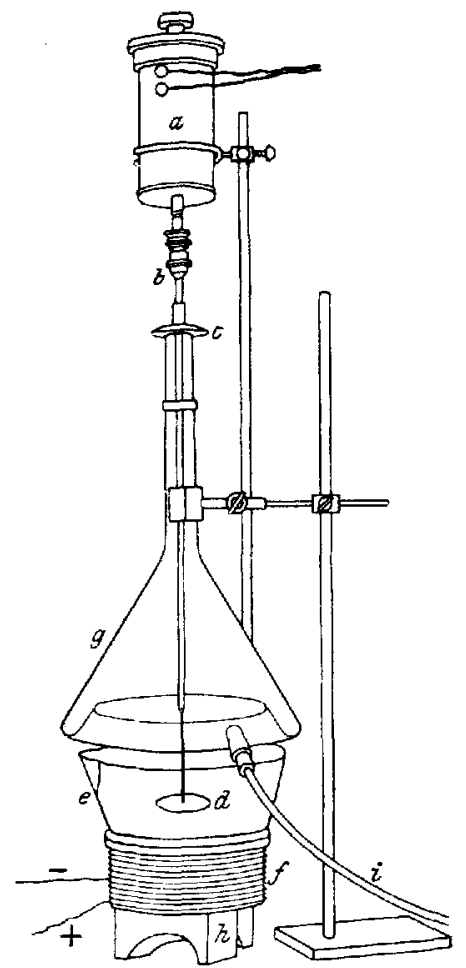

Fig. 1. sprechende Umänderung erfahren hatte (s. u.), wurde weiteres Material von hohem Reinheitsgrade hergestellt und der Gehalt an $\mathrm{Cl}^{\prime}$ darin genau bestimmt. Mit dieser Substanz wurden einige vorläufige Analysen ausgeführt.

Beim Umkristallisieren des Salzes wurde folgende elektrische Rührrorrichtung nebst Heizbad verwendet (Fig. 1).

An dem Ende eines möglichst geraden Glasstabes von ca. $40 \mathrm{~cm}$ Länge wurde mit Hilfe von Bleiglas eine gelochte, mit einem $10 \mathrm{~cm}$ langen Platinstift versehene Platinscheibe $d$, wie sie als rotierende Anode bei der Schnellelektrolyse Verwendung findet, befestigt. Der 
Stab wurde durch den Hals eines Victor Mrresschen Schutztrichters $g$ geführt und konnte bei $b$ mit Hilfe eines Elektromotors $a$ in schnelle Rotation versetzt werden. Der Hals des Trichters war so weit und die schlagende Bewegung des Glasstabes so gering, dals die Gefahr einer Verunreinigung des Chlorats durch Glassplitterchen nicht bestand. Oberbalb der Stelle, wo der Glasstab den Schutztrichter verliels, war mit Hilfe eines Stückchens Gummischlauch ein durchbohrtes Uhrglas $c$ befestigt, um das Eindringen von Staub durch die obere Öffnung des Trichters zu verhindern. Die Platinschale $e$ hatte ein Volumen von ca. $2 \mathrm{l}$. Das Heizbad $f$ bestand aus dem Porzellanuntersatz eines HACsMaN-Exsikkators (von $14 \mathrm{~cm}$ lichtem Durchmesser), der) mit zwei parallel geschalteten Widerstandsbändern von je ca. $40 \mathrm{Ohm}$ Widerstand und $0.5 \mathrm{~mm}$ Drahtstärke (von Scunıwindт-Neuenrade umwickelt war. Den Wärmeschutz nach aufsen bildete Asbestpappe. Das Ganze fand Aufstellung auf einem tönernen Dreifuls $h$, wie er im Röfslerofen als Tiegeluntersatz benutzt wird.

\section{Kaliumchlorat für die Vorversuche,}

Das Kaliumchiorat (von Kahlbaum) wurde in einem Jenaer Becher in destilliertem Wasser bei $100^{\circ}$ gelöst und die Lösung in die Platinschale filtriert. Dadurch wurden die festen Verunreinigungen des Handelssalzes entfernt. $\mathrm{Zu}$ jedem Liter Lösung wurde dann nach der Angabe von Stas, um die etwa vorhandene Kieselsäure in lösliche Form ïberzuführen, $1 \mathrm{~g}$ Kaliumbydrat ,Merck", dessen Chlorgehalt etwa $0.1 \mathrm{mg}$ betrug, hinzugefügt. Zur Ablühlung wurde die Schale unter dauerndem Rübren mit der soeben beschriebenen Vorrichtung in eine mit aus destilliertem Wasser hergestellten Eis gefüllte Porzellanschale gestellt. Hierdurch wurde einerseits die Ausbeute erböht und andererseits das Salz in feinkristallisierter Form ausgeschieden, wie es für die spätere Verwendung vorteilhaft war.

Die beiden folgenden Kristallisationen wurden nach den früheren Erfahrungen aus Lösungen von $80^{\circ}$ bewirkt, wobei jedesmal etwas Kaliumhydrat zugesetzt wurde. Die vierte Kristallisation wurde ohne Zusatz von Kaliumhydrat aus reinstem Wasser vorgenommen. Die Untersuchung ergab eiue für die Vorversuche ausreichende Reinheit der Substanz. Sie reagierte völlig neutral gegen Phenolphtalein, ihr Chloriongehalt war, wie aus folgender Tabelle hervorgeht, äufserst klein (die Bestimmungen des $\mathrm{Cl}^{\prime}$ wurden mit dem Nephelometer ausgeführt). 
Cl'-Gehalt der einzelnen Fraktionen:

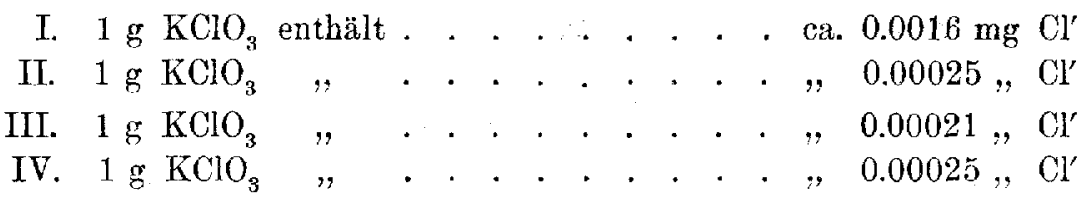

Kaliumchlorat für die Hauptversuche.

Das Ausgangsmaterial bildete in diesem Falle das reinste im Handel erhältliche Salz, "Kaliumchlorat Merck" für analytische Zwecke. Es wurden, wie bei der Darstellung des Salzes für die Vorversuche die ersten drei Kristaliisationen unter Zusatz von Kaliumbydrat, alle folgenden jedoch aus reinstem Wasser vorgenommen. Die einzelnen Fraktionen wurden auf Platintrichtern mit reinstem Wasser von $0^{\circ} \mathrm{C}$ ausgewaschen und aufserdem die Lösung vor der vorletzten Kristallisation durch ein sorgfältig ausgewaschenes, vorher mit Salzsäure, Flufssäure and Wasser behandeltes Filter gegossen, um vielleicht noch vorhandene Spuren Staub zu entfernen. Es war ursprünglich beabsichtigt, mit zwei verschiedenen Kristallfraktionen zu arbeiten. Ein Teil des Salzes wurde daher nur fünfmal, ein anderer dagegen achtmal umkristallisiert. Die erste Fraktion mufste jedoch wegen späterer zufälliger Verunreinigungen verworfen werden, so dafs zu den Hauptversuchen nur das reinste Salz (etwa $80 \mathrm{~g}$ ) zur Verfügung stand.

Nach Beendigung der Hauptversuchsreihe wurde die Substanz auf ihren Gehalt an Chlorion geprüft. Hierbei ergab sich, dafs die Substanz etwas mehr Chlorion enthielt als das bei den Vorversuchen benutzte Salz, und zwar auf jedes Gramm etwa $0.0011 \mathrm{mg}$. Nimmt man an, dafs das Kaliumchlorat zunächst nach der Gleichung

$$
2 \mathrm{KClO}_{3}=\mathrm{KClO}_{4}+\mathrm{KCl}+\mathrm{O}_{2}
$$

zerfällt, so entspricht dieser Gehalt an Chlorion einem Gewichtsverlust des Chlorats von $0.01 \mathrm{mg}$ für je $10 \mathrm{~g}$ Substanz. Diese Gewichtsänderung hat zwar auf das Endresultat einen sehr geringen Einflufs (das Atomgewicht des Chlors ändert sich hierdurch um etwa sieben Einheiten der fü nften Stelle nach dem Komma); doch wurde zur Korrektion das gefundene Gewicht des Chlorats um die angegebene Grölse (0.01 mg für je $10 \mathrm{~g}$ ) erhöht.

Die Vorversuche zur Prüfung des Kalinmchlorats auf Reinheit durch Verflüchtigung des Salzes mufsten wegen Mangels an einem geeigneten Gefälsmaterial aufgegeben werden. 
Der Schmelzpunkt des reinen Kaliumchlorats wurde zu $357^{\circ}$, das spez. Gewicht bei $18^{\circ} \mathrm{zu} 2.337$ bestimmt.

\section{Überchlorsäure.}

Käufliche verdünnte Überchlorsäure wurde zur Reinigung durch einen Platinkühler destilliert und die destillierte Säure in einem Platinfläschchen mit eingeschliffenem Platinstopfen aufbewahrt.

\section{Apparatur zur Zersetzung des Kaliumchlorats. (Siehe Figuren.)}

I. Apparate zur Herstellung, Reinigung und Trocknung der nötigen Gase.

Die Luft, die zum Trocknen des Kaliumchlorats resp. zur Wasserbestimmung, sowie zur Erzeugung des Stickstoffs für die

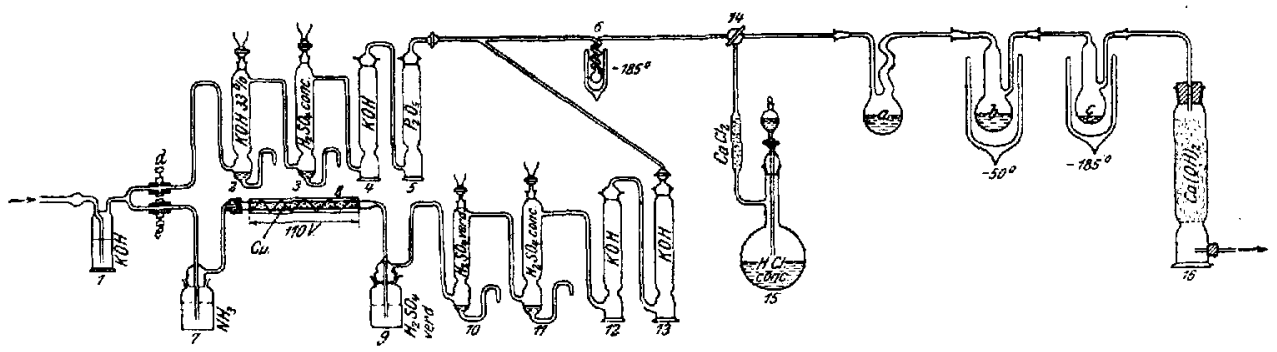

Fig. 2.

Schmelzung des Kaliumchlorids dienen sollte, wurde mit Hilfe eines Wasserstrahlgebläses eingepumpt, durch eine Waschflasche mit $33 \%$ iger Kalilauge (1) (Fig. 2) zur Entfernung des grölstenTeiles der Kohlensäure und des Wasserdampfes und dann durch ein Dreiwegstück $e d$ einerseits bei $d$ zum Trockenapparat für Luft, andererseits bei $e$ zu dem Apparat zur Erzeugung von Stickstoff nach der Methode von Lupton ${ }^{1}$ geleitet.

Der Apparat zur Trocknung der Luft bestand aus vier Türmen, von denen der erste mit Glasperlen und $33 \%$ iger Kalilauge (2), der zweite mit Glasperlen und konzentrierter Schwefelsäure (3), der dritte mit Kaliumhydrat in Stücken (4) und schliefslich der vierte mit Glasperien und reinstem Phosphorpentoxyd ,Merck“(5) gefüllt war. Das Gasleitungsrohr vereinigte sich dann wieder mit den für den Stickstoff bestimmten und führte zu einer Glasspirale (6), die in flüssige Luft tauchte. Diese letztere Vorsichtsmafsregel wurde

${ }^{1}$ Chem. News 3a (1876), 90. 
angewendet, um alle etwa durch die Trockenmittel verursachten Verunreinigungen der Luft, wie z. B. von der konzentrierten Schwefelsäure Spuren Schwefeldioxyd oder vom Phosphorpentoxyd Spuren Phosphorwasserstoff, sicher zurückzuhaiten. Bei diesem Apparat waren alle Verbindungen soweit als angängig durch Verschmelzen, sonst durch Glasschliffe hergestellt.

Der Apparat zur Erzeugung des Stickstoffes bestand aus einer Waschflasche mit konzentriertem wässerigen Ammoniak (7), dann folgte ein schräg nach unten gerichtetes, elektrisch geheiztes Verbrennungsrohr (8), das mit Kupferstückchen gefüllt war, daran schlossen sich eine Waschflasche mit verdünnter Schwefelsäure $(9)$ (mit etwas Methylorange) zur Absorption des überschüssigen Ammoniaks an, und schliefslich ein ähnlicher Trockenapparat, wie für die Luft. Die vier Türme enthielten hier verdünnte Schwefelsäure(10), konzentrierte Schwefelsäure (11) und Kaliumbydrat in Stücken (12 u. 13). Hier waren die Verbindungen teilweise durch Gummischlauch hergestellt, doch so, dafs die Glasteile übereinandergriffen oder sich berührten. Jeder der beiden Trockenapparate konnte, ehe sich die Gaswege wieder trafen, durch Glashähne abgeschlossen werden. Hinter der durch flüssige Luft gekühlten Glasspirale war ein Dreiweghahn (14) angebracht, durch den die Gasleitung mit einem Chlorwasserstoffentwickler (15) in Verbindung gesetzt werden konnte.

Der Chlorwasserstoff wurde dargestellt, indem man in einer Gasentwickelungsflasche (15) mit eingeschliffenem Tropftrichter konzentrierte Salzsäure (spez. Gew. 1.19) gelinde erwärmte. Diese Salzsäure wurde, um sie von Wasser und mitgerissenen Flüssigkeitströpfchen zu befreien, durch ein kleines, in die Gasleitung eingeschmolzenes Chlorcalciumrohr geleitet. Derartig dargestelltes Chlorwasserstoffgas ist völlig rein, wie von RICHARDs und $W_{E L L S}{ }^{1}$ gezeigt worden ist. Die notwendigen Verbindungen waren durch gute, ungefettete Schliffe hergestellt.

Hinter dem Dreiweghahn endete die Gasleitung in einem mit besonderer Sorgfalt hergestellten Schliffe, an welchem der eigentliche Zersetzungsapparat $a$ angesetzt werden konnte.

II. Beschreibung des Zersetzungsgefälses.

Das Zersetzungsgefäfs ( $a$ in Fig. 2 u. 3$)^{2}{ }^{2}$ dessen endgültige Form erst nach vielen Vorversuchen gewählt wurde, bestand aus einem

' Kichards, Untersuchungen über Atomgewichte, S. 698-699.

${ }^{2}$ In Fig. 2 ist der Mafsstab der Gefälse $a, b$ und $c$ verdoppelt. 
Quarzglaskölbchen a von ca. $45 \mathrm{~mm}$ Durchmesser mit $\mathrm{Zu}-$ und Ableitungsröhren, deren Gestalt und Abmessung aus Fig. 3 a ersichtlich ist. Der Boden war, um das Gefäls gegen die beim Erstarren der geschmolzenen Salze auftretenden Kräfte widerstandsfähiger zu

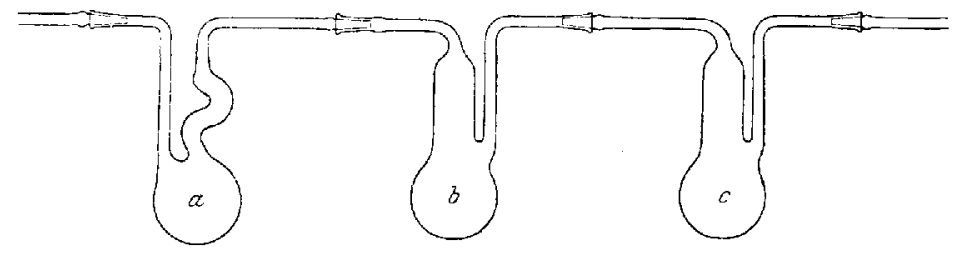

Fig. 3.

machen, verstärkt. $\mathrm{Zu}$ diesem Gefäls war ein genau gleiches als Taragefäls vorhanden, dessen Gewichtsdifferenz gegen das erste durch Quarzstückchen ausgeglichen war. Zum Verschlul's während der Wägung dienten Glasstöpsel und Glaskappe. An das Zersetzungsgefäfs schlossen sichzur Feststellung der bei der Reaktion übergerissenen Substanzmenge zwei weitere Gefäfse ( $b$ u. $c$ ) von der aus der Abbildung ersichtlichen Form an. Fon diesen war das erste $(b)$ aus Quarzglas, das zweite $(c)$ anfänglich aus Jenaer Glas, später jedoch, da dies notwendig wurde, ebenfalls aus Quarzglas hergestellt. Fon hier aus ging mittels Glasschliffs die Gasleitung zu einem Turm mit gelöschtem Kalk (16), um die Chloroxyde resp. das Chlor unschädlich zu machen, und schliefslich ins Freie.

Zur Apparatur gehörten aufserdem noch ein elektrisch heizbarer (Platindraht-)Tiegelofen, mit Regulierwiderstand und zwei Dewarzylindern für die Vorlagen.

III. Das Phosphorpentoxydrohr zur Wasserbestimmung.

Da zur Ermittelung des genauen Gewichtes des Chlorats eine exakte Wasserbestimmung notwendig war, so wurden zwei Phosphor$\lessdot$ pentoxydröhrchen mit Glashähnen, von denen das eine als Tara diente, beschafit. Das zur Bestimmung verwandte Röhrchen hatte nebenstehende Gestalt (Fig. 4). Die angewandte Länge der Phosphorpentoxydschicht reicht nach den Angaben ron MoRLEX ${ }^{1}$ dazu aus, keine wägbare Menge Wasser Fig. 4. entweichen zu lassen. Der Inhalt des Röhrchens (') vatürl. Größse)

${ }^{1}$ Am. Journ. Soe. 3, 33. 199; Zeitschr. analyt. Chem. 3 (1864), 407; Journ. Am. Chem. Soc. 26 (1904), 1171; Z. B. 2 (1904), 1366. 
bestand bei den Vorbestimmungen aus reinstem käuflichen Phosphorpentoxyd, gemischt mit reinstem Asbest, wie er zur Füllung von Goochtiegeln dient. Für die Hauptbestimmungen wurde das verwandte Phosphorpentoxyd über Platinasbest einer erneuten Sublimation im Sauerstoffstrome unterworfen.

\section{Ausführung der Bestimmnng.}

I. Wägung.

Zur Wägung wurde eine Wage von der Firma Fleischer und Mentz mit vergoldeten Balken benutzt, die bei einer Belastung von ca. $40 \mathrm{~g}$ noch für $0.02 \mathrm{mg}$ Übergewicht einen deutlichen Ausschlag gab. Ein $5 \mathrm{mg}$ Platinreiter gestattete am Lineal bequem die Schätzung der Hundertstel Milligramme auf eine Einheit. Alle Wägungen wurden nach der Substitutionsmethode ${ }^{1}$ ausgeführt. Der Ausgleich der zur Wägung und Substitution dienenden Gefälse wurde bis auf einige Zehntel Milligramme gebracht, der Gewichtssatz auf das genaueste geeicht. Die Durchrechnung für die Vakuumkorrektur ergab die Notwendigkeit, Luftfeuchtigkeit, Luftdruck und Temperatur während der Wägung zu beobachten.

Die folgende Rechnung gibt einen Begriff von der Gröfse der letzteren beiden Korrekturen. Beobachtet wurden Schwankungen innerhalb dreier Tage (der Zeit, die verläuft von der Wägung des $\mathrm{KClO}_{3}$ bis zur Wägung des $\mathrm{KCl}$ ).

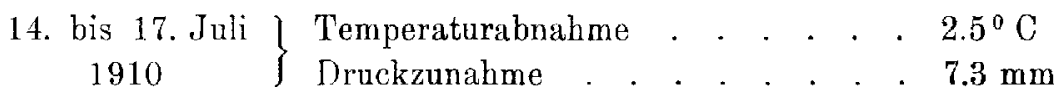

I. Spez. Gew. der Luft bei $21^{\circ} \mathrm{C}$ und $760 \mathrm{~mm}$. . . 0.001201 II. " $\quad, \quad, \quad, \quad 18.5^{\circ} \mathrm{C}, 767, \quad$. . . 0.001222

Die wirklichen Drucke und Temperaturen waren hiervon etwas verschieden. Die obigen Zahlen wurden zur Erleichterung der Rechnung gewählt, sie sollen nur als Beispiel dienen.

Vakuumfaktor für $\mathrm{KClO}_{3}$ zu I. 1.0003709

$$
" \quad . \quad \mathrm{KClO}_{3} \text { zu II . } 1.0003774
$$

Das heifst: $10 \mathrm{~g} \mathrm{KClO}_{3}$ in Luft gewogen geben:

$$
\begin{aligned}
& \text { bei I } 10.00371 \mathrm{~g} \mathrm{KClO}_{3} \text { absolut, } \\
& \text { bei II } 10.00377 \mathrm{~g} \mathrm{KClO}_{3}, ",
\end{aligned}
$$

also eine Differenz von: $0.00006 \mathrm{~g}$,

1 Vgl. Ricrands, Z. anorg. Chem. 3 (1893), 445; 10 (1895), 19.

Z. anorg. Chem. Bd. 71. 
eine Grölse, die durch Wägung leicht feststellbar ist, und daher nicht vernachlässigt werden darf.

Für $\mathrm{KCl}$ ist die Differenz, auf $10 \mathrm{~g}$ berechnet (entsprechend dem kleineren spezifischen Gewicht), etwas grölser, ca. $0.00007 \mathrm{~g}$.

Die zu wägenden Apparate bestanden zum grölsten Teil aus Quarzglas und zeigten daher die bekannte Gewichtskonstanz, die diese Apparate vor solchen aus gewöhnlichem Glase auszeichnet. Die anfänglich durch elektrische Ladungen auftretenden Schwierigkeiten konnten durch die Verwendung stark radioaktiver Präparate, für deren freundliche Überlassung wir Herrn Professor Dr. O. HAHN ganz besonderen Dank schulden, leicht beseitigt werden. Der Feuchtigkeitsgehalt im Wägezimmer war stets so gering, dals sich daraus keine Schwierigkeiten ergaben.

\section{Wasserbestimmung.}

In einem reinen Platinschälchen wurden ca. $10-12 \mathrm{~g}$ exsikkatortrockenes Kaliumchlorat annähernd abgewogen und mit einem Platintiegel, der ebenso wie das Schälchen zu keinem anderen Zwecke verwendet wurde, zerdrückt, so dafs keine Klümpchen mehr vorhanden waren. Dann wurde mittels eines trockenen, abgesprengten Glastrichterchens durch das gerade Gaszuführungsrohr des Zersetzungsgefälses, dessen Übergewicht über das als Tara dienende Gefäls vorher genau festgestellt war, das Kaliumchlorat in dieses eingebracht. Der Schliff wurde sodann mittels eines trockenen und staubfreien Pinselchens von etwa haften gebliebenem Kaliumchlorat sorgfältigst gereinigt.

Bei den Vor- und Hauptbestimmungen wurde bei der nun folgenden Wasserbestimmung in etwas verschiedener Weise gearbeitet.

Bei den Vorbestimmungen wurde wie folgt verfahren:

Das Gefäfs wurde, mit einer Platinschlinge an einem Glashaken hängend, an den Schliff des Lufttrockenapparates angesetzt und bei Zimmertemperatur zur Konstanz getrocknet. Den Abschluls gegen die Atmosphäre bildeten ein Phosphorpentoxyd- und zwei Chlorcalciumröhrchen. Gewichtskonstanz war stets eingetreten, wenn die vorher sorgfältigst im Exsikkator über Kaliumbydrat getrocknete Substanz ca. 1 Stunde im Luftstrom behandelt worden war. Nach der Wägung wurde dann zur Wasserbestimmung geschritten, wozu das Gefäls wieder an den Lufttrockenapparat angesetzt wurde. Der Schliff hielt zwar einem Überdruck von einigen Zentimetern Wasser- 
säule ohne weiteres Widerstand, trotzdem wurde über die Verbindungsteile ein Stückchen reinen ausgekochten und röllig trockenen Kapillarschlauches gezogen. Nachdem nun etwa 5 Minuten Luft durch den Apparat geleitet war, wurde das Phosphorpentoxydrohr mittels Kapillarschlauches an dem Gefäls befestigt. Den Abschlufs gegen die Zimmerluft bildeten wie vorher ein Phosphorpentoxydund zwei Chlorcalciumröhrchen. Das Zersetzungsgefäls wurde jetzt mittels des elektrischen Tiegelofens soweit erhitzt, dals die Luft. temperatur im Ofen ca. $420^{\circ}$ betrug. War die dadurch erzielte Schmelzung des Chlorats vollendet, so wurde der Ofen entfernt, das Gefäls, um ein Zerspringen zu verhindern, während des Erstarrens des Salzes gelinde hin- und hergeschwenkt und schliefslich noch $1 \frac{1}{2}$ Stunden Luft durch den Apparat geleitet.

Ein blinder Vorversuch hatte ergeben, dafs das Phosphorpentoxydrohr, wenn der Apparat durch vorheriges Durchleiten von Luft völlig ausgetrocknet war, in dieser Zeit keine wägbare Wassermenge aufnahm.

Bei der Schlufsreihe wurde nicht wie bei den Vorversuchen zur Konstanz getrocknet, sondern das exsikkatortrockene Salz direkt gewogen, das Phosphorpentoxydröhrchen sofort nach dem Entfernen der Verschlufskappe mittels eines Stückchens Kapillarschlauch an das Zersetzungsgefäls angesetzt, und das Gefäls wie vorher am Lufttrockenapparat angebracht. Die Wasserbestimmung selbst wurde wie bei den Vorversuchen weitergeführt.

Als besondere Vorsichtsmalsregel wurde, um einen zu hohen Wert der Wasserbestimmung zu vermeiden, vor jeder Bestimmung bei den Hauptrersuchen der ganze Trockenapparat samt seinen Gasleitungen mit einem durch Phosphorpentoxyd getrockneten Luftstrome 15-20 Stunden lang behandelt.

\section{Die quantitative Reduktion des Kaliumchlorats zu Kaliumchlorid.}

Nach beendeter Wasserbestimmung wurde das Phosphorpentoxydrohr abgenommen, das im Zersetzungsgefäls befindliche geschmolzene Chlorat mit der zur Lösung des bei der Zersetzung entstehenden Chlorids nötigen Menge Wasser übergossen, die Vorlagen angesetzt und diese auf die nötige Temperatur abgekühlt $\left(-60\right.$ bis $-50^{\circ}$ in $b$ und ca. $-185^{\circ}$ in $\left.c\right)$. Dann wurde unter Kühlung des Zersetzungsgefälses mit reinstem Wasser von $0^{0}$, das sich bei den Vorbestimmungen in einem Jenaer Becherglas, bei den Haupt- 
bestimmungen in einem vergoldeten Becher aus chemisch-reinem Silber befand, Salzsäuregas eingeleitet, bis in der Kälte die Gasentwickelung eben begann. Bei den Vorbestimmungen stellte sich ein weiteres Einleiten von Salzsäuregas während der Zersetzung als ungünstig heraus, einerseits, weil ziemliche Mengen Chlorwasserstoff, die bei der hohen Temperatur, die sich durch die Reaktionswärme einstellt, nicht absorbiert werden, in der Jetzten Vorlage erstarrten und diese auszufüllen drohten, andererseits aber auch die durch den Gasstrom mitgerissene Substanzmenge ganz unverhältnismäfsig anstieg. Das Kühlbad wurde nach Beginn der Reaktion entfernt, worauf die Zersetzung des Chlorats unter Erwärmung der Flüssigkeit von selbst weiterging; zum Schlufs wurde so lange erhitzt, bis die Gasentwickelung aufhörte, was bei beginnendem Sieden der Fall war. Die noch schwach gelb gefärbte Flüssigkeit wurde nun wieder auf nahezu $0^{\circ}$ abgekühlt, von neuem Salzsäuregas bis zur beginnenden Reaktion eingeleitet und wiederum wie vorher verfahren. Diese Behandlung wurde so oft wiederholt, bis zum Schluls in der Hitze eine klare, farblose Lösung von Kaliumchlorid übrig blieb und auf erneutes Einleiten von Salzsäuregas keine Reaktion oder Gelbfärbung der Flüssigkeit mehr eintrat. Ein zu grofser Überschufs von Salz. säure, der die vollständige Lösung (s. o.) des Kaliumchlorids verhindert hätte, konnte bei vorsichtiger Beendigung des Prozesses stets vermieden werden.

Das während der Reaktion entweichende Gemisch von Chloroxyden und Chlor wurde in der Vorlage $b$ durch ein Gemisch ron Alkohol und flüssiger Luft bei ca. -50 bis $-60^{\circ}$ völlig kondensiert; etwa doch von der Salzsäure mitgerissene Anteile und diese selber wurden in der zweiten Vorlage $c$, die direkt mit flüssiger Luft gekühlt wurde, zum Erstarren gebracht, so dafs während der Zersetzung keine merklichen Gasmengen aus dern Zersetzungsapparat entwichen und daher alle vom Gasstrom mitgerissenen Salzteilchen aufgefangen wurden. War die Reaktion beendet, so wurde das Zersetzungsgefäls bis nahe an den Siedepunkt der salzsäurehaltigen Kaliumchloridlösung erhitzt und die Flüssigkeit durch Überleiten von trockener Luft, in der Regel über Nacht, langsam abgedunstet. Die hierbei überdestillierende wässerige Salzsäure kondensierte sich dann in der jetzt auf Zimmertemperatur befindlichen ersten und auch spurenweise in der zweiten Vorlage. Das Salz im Zersetzungsgefäls wurde noch einige Zeit auf $200-300^{\circ}$ erhitzt, damit das Wasser nach Möglichkeit entfernt würde, und schliefslich in eines 
Stickstoffatmosphäre, nicht im Stickstoffstrom, der eventuell verflüchtigtes Kaliumchlorid hätte entführen können, zur Entfernung des letzten Restes des Wassers und vor allem der eingeschlossenen Salzsäure geschmolzen. Schlielslich wurde im trockenen Luftstrom erkalten gelassen, der Apparat auseinandergenommen, die Glasverschlüsse auf das GefäB a aufgesetzt und letzteres nach mehrstündigem Stehenlassen im Wägezimmer gewogen.

IV. Bestimmung des Inhaltes der Vorlagen.

Der durch Chlor und Chloroxyde rotgelb getärbte Inhalt der Vorlagen $b$ und $c$, - auch der zweiten, nachdem diese aus Quarzglas hergestellt war (s. o.), da die zuerst beabsichtigte nephelometrische Bestimmung des Inhaltes dieser Vorlage aus gleich zu erörternden Gründen nicht angängig war -- wurde mit reinstem Wasser vorsichtig in eine Quarzschale gespült und Iangsam auf einem elektrisch geheizten Luftbade abgedunstet. Die hierbei zurückbleibende äufserst geringe Salzmenge wurde, in wenigen Kubikzentimetern reinstem Wasser gelöst, in ein kleines Quarztiegelchen gespült, mit einigen Tropfen Überchlorsäure zur 'Trockne gedampft, bei ca. $300^{\circ}$ getrocknet und nach dem Erkalten gewogen, wobei ein zweites Quarztiegelchen als Substitutionsgefäfs diente.

Dieses Abdampfen mit Überchlorsäure und die Umrechnung der Korrektur auf Kaliumchlorid aus dem so gefundenen Gewicht von Kaliumperchlorat war der Ausweg aus einer Schwierigkeit, die sich bei der Ausführung der Analysen herausstellte. Die mitgerissene Substanzmenge schien nämlich - wenigstens bei den Versuchen, bei denen mit dauerndem Salzsäurestrom gearbeitet wurde in Anbetracht der Behauptung von Stas, dals keine Substanz bei seinen Versuchen mitgerissen worden wäre, etwas grols zu sein. Andererseits aber wollten wir auch, trotzdem man von vornherein eigentlich nur Kaliumchlorid hätte erwarten sollen, uns doch durch eine nephelometrische Probe von der Richtigkeit dieser Vermutung überzengen. Die Untersuchung zeigte indessen, dals die gewogene Salzmenge der Vorlagen nur zum allergeringsten Teile a us Kaliumchlorid bestand. Die nun folgende, recht mühsame Arbeit ergab, dals, wie das schon von anderer Seite beobachtet worden war, beim Abdunsten wässeriger Lösungen von Chloroxyden am Licht Überchlorsäure entsteht, und dafs das von uns gewogene Salz daher z. T. Kaliumperchlorat war, welches durch das bei den ersten Versuchen bei zu hoher Temperatur erfolgte Konstantmachen 
sich spurenweise zersetzt hatte. Es stellte sich als nicht angängig heraus, dieses Kaliumperchlorat zu reduzieren, da das einzige dazu mögliche Verfahren, das Abrauchen mit Ammoniumchlorid Verluste an Kaliumchlorid herbeigefübrt hätte. Das Salz wurde daher durch Zugabe einiger Tropfen reinster Überchlorsäure quantitativ in Kaliumperchlorat verwandelt. Dieses Verfahren ist nicht nur nicht fehlerhaft, sondern sogar für die Bestimmung der Korrektur günstig. Da die Korrektur als Perchlorat bei vorsichtiger Leitung der Zersetzung nur einige Zehntel Milligramm beträgt, die Wägegenauigkeit dieser kleinen Menge aber nur etwa $5 \%$ ist, das zurzeit angenommene Verhältnis $\mathrm{KClO}_{4} / \mathrm{KCl}$ aber mindestens die 100 -facbe Genauigkeit hat, so ist der durch die Umrechnung bedingte Fehler im Gewicht des Kaliumchlorids unwägbar klein. Man hat dagegen den Vorteil, dafs wirklich vorhandene Wägefehler oder auch spurenweise Verunreinigungen entsprechend dem Verhältnis $\mathrm{KClO}_{4} / \mathrm{KCl}$ weniger auf das Resultat Einflufs haben.

\section{Vorläufige Ergebnisse.}

Die folgende Tabelle gibt Aufschlufs über die gefundenen vorläufigen Werte des Verhältnisses $\mathrm{KClO}_{3} / \mathrm{KCl}$.

Von den übersprungenen Nummern diente Versuch III zur Untersuchung der Korrektursubstanz, IV konnte nicht beendet werden.

\begin{tabular}{c|c|c|c|c}
\hline \hline $\begin{array}{c}\text { Vor- } \\
\text { versuch- } \\
\mathrm{Nr}\end{array}$ & $\begin{array}{c}\text { Absol. Gewicht des } \\
\text { angew. KClO } \\
\text { (wasserfrei) in g }\end{array}$ & $\begin{array}{c}\text { Absol. Gew. } \\
\text { des gef. KCl } \\
\text { in g }\end{array}$ & $\begin{array}{c}\mathrm{KClO}_{3} \\
\mathrm{KCl} \\
\mathrm{ing}\end{array}$ & $\begin{array}{c}\text { Gewichtsabnahme } \\
\text { des Zersetzungs- } \\
\text { gefüfses in mg }\end{array}$ \\
\hline \hline I & 12.38248 & 7.53218 & 1.643943 & 0.36 \\
II & 11.28213 & 6.86340 & 1.643811 & 0.23 \\
V & 14.22480 & 8.65366 & 1.643791 & 0.75 \\
VI & 11.52268 & 7.00963 & 1.643835 & 0.28 \\
VII & 12.44913 & 7.57331 & 1.643816 & 0.54 \\
\hline
\end{tabular}

Mittel aus Vers. II-VII: 1.643813

Für $O=16.000$ und $\frac{\mathrm{K}}{\overline{\mathrm{Cl}}}=1.102641$ (RICHARds und StäHLER ${ }^{1}$ ) ergibt sich:

$$
\mathrm{KCl}=74.5558 \quad \mathrm{~K}=39.0976 \quad \mathrm{Cl}=35.4582
$$

Bestimmung $I$ ist nur der Vollständigkeit halber in die Tabelle aufgenommen, sie ist fehlerhaft, da wegen Mangels an flüssiger

${ }^{1}$ Journ. Amer. Chem. Soc. 29 (1907), 623-639; Ber. deutsch. Chem. Ges. 39 1906), $3611-3625$. 
Luft die entweichenden Gase nicht völlig kondensiert werden konnten. Aufserdem wurde die Zersetzung des Chlorats im lebhaften Salzsäurestrom ausgeführt und das Chlorid im Stickstoffstrom geschmolzen, so dafs die Verluste an Kaliumchlorid bei diesem Versuche erklärlich sind.

Die Übereinstimmung der für das Verhältnis $\mathrm{KClO}_{3} / \mathrm{KCl}$ gefundenen Werte ist, wie die Tabelle zeigt, eine recht befriedigende. Sie gab uns zugleich die Gewifsheit, dafs wir auch den letzten Einwand, den man gegen die Richtigkeit des gefundenen Resultates noch erheben kann, überwinden würden.

Das Gefäls $a$ war nach den ersten etwa zehn hier nicht angegebenen Vorbestimmungen völlig gewichtskonstant. Später zeigte es jedoch nach dem Schmelzen des Kaliumchlorids eine zwar geringe, aber nicht zu vernachlässigende Gewichtsabnahme. In obiger Tabelle sind die Resultate obne Berücksichtigung dieser Differenzen berechnet. Trotzdem weichen sie um nur etwas mehr als zwei Einheiten in der fünften Dezimale vom Mittelwert ab. Daraus geht hervor, dals eine Beeinflussung der Werte durch die Gewichtsabnahme des Gefälses nicht möglich ist, da schon ein Zehntel Milligramm Gewichtsänderung des Kaliumchlorids bei der angewandten Substanzmenge eine Änderung des Verhältnisses $\mathrm{KClO}_{3} / \mathrm{KCl}$ um ebenfalls zwei Einheiten der fünften Dezimale herbeigeführt haben würde. Eine Verflüchtigung von Siliciumdioxyd durch Umwandlung in Siliciumchlorid war äufserst unwahrscheinlich, zumal sich dann die Kieselsäure in den Vorlagen hätte finden müssen. Eine Bildung ron Kaliumsilikat war nicht nachweisbar, da die Lösung des Kaliumchlorids völlig neutral reagierte. Es blieb daher nur die Vermutung übrig, dås das Kaliumchlorid beim Erstarren von den Gefälswänden mechanisch Kieselsäure abrifs, die nachher beim Entleeren des Gefäfses mit entfernt wurde. Das Gefäfs war nämlich bei einigen Versuchen so weit überhitzt worden, dafs im Quarzglas Tridymitbildung eingetreten war und die Innenwand eine zunehmend rauhere, etwas blätternde Oberfläche angenommen hatte, die leicht Quarzteilchen mechanisch an das erstarrende Salz abgeben konnte. In der Tat wurde das Gefäls beim Erstarren des Kaliumchlorids sehr stark beansprucht, was daraus hervorging, dafs es hierbei einmal zersprang. Für diese Annahme sprach auch die Tatsache, dals bei Versuch $V$ die Gewichtsabnahme des Gefälses am grölsten ist, bei dem das Kaliumchlorid zweimal geschmolzen wurde, also auch eine doppelte Menge Kieselsäure von der Gefälswand abge- 
trennt werden mulste. Das Gewicht, Apparat plus Kaliumchlorid, war jedoch, was die obige Vermutung über die Art der Gewichtsabnahme des Gefälses noch bestärkte, durch die zweite Schmelzung nicht im geringsten verändert worden.

\section{Endgültige Ergebnisse.}

Um die Frage über die Gewichtskonstanz des Gefärses während der Operation einwandfrei beantworten zu können, liefsen wir für die letzten Versuche ein neues Gefäfs anfertigen, bei dem besonders darauf geachtet wurde, dafs die Innenwand vollkommen glatt und glasklar war.

Einige blinde Versuche, mit einem etwas weniger reinen Kaliumchlorat, ergaben in Übereinstimmung mit den allerersten Versuchen eine ausgezeichnete Gewichtskonstanz dieses neuen Gefäfses.

Es wurde daher sofort mit der endgültigen Versuchsreihe begonnen, deren Resultate die untenstehende Tabelle wiedergibt.

\begin{tabular}{|c|c|c|c|c|}
\hline $\begin{array}{c}\text { Analysen- } \\
\text { Nr. }\end{array}$ & $\begin{array}{l}\text { Absol. Gew. des } \\
\text { angew. } \mathrm{KClO}_{3} \\
\text { (wasserfrei) in } \mathrm{g}\end{array}$ & $\begin{array}{l}\text { Absol. Gew. } \\
\text { des gef. } \mathrm{KCl} \\
\text { in } \mathrm{g}\end{array}$ & $\begin{array}{l}\text { Übergewicht d. Zer- } \\
\text { setzungsgefäfses üb. } \\
\text { die Tara in mg }\end{array}$ & $\frac{\mathrm{KClO}_{\mathrm{B}}}{\mathrm{KCl}}$ \\
\hline I & 10.26355 & 6.24370 & 1.20 & 1.643824 \\
\hline II & 10.08261 & 6.13362 & 1.22 & 1.643826 \\
\hline III & 10.03177 & 6.10269 & 1.22 & 1.643828 \\
\hline IV & 10.63651 & 6.47073 & 1.20 & 1.643786 \\
\hline V & 12.05095 & 7.33096 & 1.18 & 1.643842 \\
\hline
\end{tabular}

Für $0=16.000$ und $\frac{\mathrm{K}}{\mathrm{Cl}}=1.102641$ (RICHARds und STÄHLER) ergibt sich:

$$
\mathrm{KCl}=74.5551 \quad \mathrm{~K}=39.0973 \quad \mathrm{Cl}=35.4578 .
$$

\section{Kritik der Ergebnisse.}

In der vorliegenden Arbeit sind einige Fehler früherer Experimentatoren aufgefunden und bei der Neubestimmung vermieden worden, so dafs konstante Fehler, soweit dies augenblicklich zu beurteilen ist, in dem Endresultat nicht mehr vorhanden sind. Die einzigen Möglichkeiten, die noch eine Änderung der gefundenen Werte bewirken könnten, wären eine etwa rorhandene Spur Kiesel- 
säure oder winzige Mengen Staub, die während des Arbeitens trotz aller Vorsicht in das Chlorat gekommen sein könnten.

Durch Kieselsäure, wie überhaupt jede nicht flüchtige Verunreinigung, würde das Verhältnis $\mathrm{KClO}_{3} / \mathrm{KCl}$ zu klein erscheinen, also die Atomgewichte von Kalium und Chlor zu grofs gefunden werden, während Staub, soweit er organischer Natur, also flüchtig, ist, die entgegengesetzte Änderung des Resultates bewirken würde.

In Anbetracht der grofsen Zahl von Kristallisationen, bei denen nur in Platingefälsen gearbeitet wurde, und bei der Sorgfalt, mit der die Substanz vor Staub geschützt wurde, ist der Einflufs dieser beiden noch möglichen Korrekturen sicher verschwindend klein.

Man kann daher annehmen, dals für

$$
\mathrm{O}=16 \text { und } \frac{\mathrm{K}}{\mathrm{Cl}}=1.102641
$$

die Atomgewichte des Kaliums und Chlors die Werte haben:

$$
\begin{aligned}
& \mathrm{K}=39.097+0.0004 \\
& \mathrm{Cl}=35.458 \pm 0.0004
\end{aligned}
$$

\section{Zusammenfassung,}

Die Ergebnisse der vorliegenden Arbeit lassen sich in folgende Punkte zusammenfassen:

Es wurde

1. gezeigt, dals Kaliumchlorat in einer für Atomgewichtsbestimmungen genügend reinen Form herstellbar ist.

2. gezeigt, dafs Kaliumchlorat nicht, wie Stas angegeben hat, unzersetzt schmelzbar ist.

3. eine Methode zur exakten Wägung des Kaliumchlorats und anderer sich beim Schmelzen ähnlich verhaltender Stoffe angegeben.

4. durch Verwendung von Quarz- und Platingefälsen der Einschlufs von Kieselsäure in die angewandten Salze vermieden.

5. eine Methode angegeben, um die von einem kondensierbaren Gasstrom mitgeführte feste Substanzmenge quantitativ zurückzuerhalten.

6. durch Einwirkung von Salzsäure Kaliumchlorat in Kaliumchlorid verwandelt und das genaue Verhältnis der Molekulargewichte dieser beiden Stoffe bestimmt. 
Vorstehende Untersuchung wurde mit besonderer Unterstützung des $\mathrm{Kgl}$. preufsischen Kultusministeriums, welches die Mittel zur Beschaffung ron Platin- und Quarzgeräten, sowie der elektrischen Heizöfen bewilligt hat, ausgeführt. Diesem sowie den Herren Sr. Exzellenz Prof. Dr. Emr Fischer und Prof. Dr. T. W. Rrchards, die unserer Arbeit Förderung und Anregung gebracht haben, soll auch an dieser Stelle herzlichster Dank gezollt werden.

Berlin, Chemisches Institut der Lniversität.

Bei der Redaktion eingegangen am 3. Juni 1911. 